\title{
Evidence for intranasal oxytocin delivery to the brain: recent advances and future perspectives
}

\author{
Daniel S Quintana1, Knut T Smerud², Ole A Andreassen ${ }^{1}$ \& Per G Djupesland*,3 \\ ${ }^{1}$ NORMENT, KG Jebsen Centre for Psychosis Research, Division of Mental Health \& Addiction, University of Oslo, \& Oslo University \\ Hospital, Oslo, Norway \\ ${ }^{2}$ Smerud Medical Research International AS, Oslo, Norway \\ ${ }^{3}$ OptiNose AS, Oslo, Norway \\ *Author for correspondence: per.djupesland@optinose.com
}

The neuropeptide oxytocin plays an evolutionarily conserved role in mammalian social behavior. Despite striking effects on animal social behavior after intracerebroventricular drug delivery, this delivery mode is impractical in humans. Intranasal oxytocin delivery provides a noninvasive alternative to increase central oxytocin activity, and has shown promise as a treatment for psychiatric illnesses. Intranasal oxytocin delivery is purported to increase central oxytocin concentrations via channels surrounding trigeminal and olfactory nerve fibers, which may facilitate increased activity at central oxytocin receptors. This report outlines the evidence for intranasal oxytocin delivery increasing central concentrations or activity, identifies current knowledge gaps and highlights future research opportunities. Recent efforts to enhance intranasal oxytocin delivery via improved intranasal delivery technology and dose-ranging studies are discussed.

First draft submitted: 4 January 2018; Accepted for publication: 29 May 2018; Published online:

26 June 2018

Keywords: drug delivery • intranasal • neuropeptide • oxytocin • psychiatry • social behavior

Oxytocin is a neuropeptide known for its role in uterine contraction during labor and milk ejection during nursing. However, emerging work has revealed its role in centrally mediated social behaviors. Since Kosfeld and colleagues [1] first reported that intranasal oxytocin increases trust in humans over a decade ago [2], there has been a surge of publications reporting its effects on human social cognition and behavior. For example, research indicates that intranasal oxytocin administration improves theory of mind, memory for social cues, and increases gaze to the eye region [3]. These findings, among others, have sparked interest in the use of intranasal oxytocin to treat psychiatric disorders characterized by social dysfunction; however, meta-analyses of oxytocin's effect in clinical populations have revealed mixed results to date $[4,5]$.

A central premise of oxytocin research is that intranasally administered oxytocin reaches the central nervous system and increases central concentrations of oxytocin allowing increased activity at central oxytocin receptors. This transport is purported to occur via ensheathed channels surrounding olfactory and trigeminal nerve fibers after nasal spray deposition onto the olfactory and respiratory epithelia. The purpose of this article is to outline the evidence for intranasal oxytocin delivery to the brain. It is hoped that by encouraging a better understanding of this evidence, researchers will recognize and pursue the potential advantages of intranasal transport to improve central delivery and ultimately improve treatment of brain diseases. We will also cover recent efforts to improve nose-to-brain delivery and offer future perspectives for the field.

\section{Direct oxytocin delivery to the brain modulates social behavior in rodents}

Oxytocin's cognitive and behavioral effects have been largely attributed to central oxytocin receptor activity [6]. The blood-brain barrier (BBB), incorporating capillary endothelium with high resistance tight junctions between the cells, forms a central barrier preventing drugs from entering brain tissues. As peripherally administered oxytocin 
is thought to enter the central compartment across the BBB only in very small amounts after substantial single doses [7], researchers have used direct oxytocin delivery to the brain to study its biobehavioral effects in animals. There is a large body of animal research reporting that intracerebroventricular (ICV) oxytocin delivery, which is thought to diffuse oxytocin throughout the brain [8], modulates social behavior, although ICV delivered oxytocin may not necessarily reach all regions equivalently. For instance, ICV administration of oxytocin facilitates partner preference in prairie voles [9], induces maternal behavior in virgin female rats [10], and facilitates social memory in rodents [11-13]. More recent work has administered oxytocin to specific brain regions in animals. For example, oxytocin injection into the medial amygdala of mice lacking the oxytocin gene rescues social amnesia [14]. Injection into the central amygdala in rats has also been reported to have anxiolytic [15] and antiaggressive effects [16]. Conversely, oxytocin antagonists block social behavior [14], highlighting the critical role of the oxytocin system.

\section{Translating animal research to human trials}

As oxytocin is an evolutionarily conserved system, researchers have investigated if these social effects found in animals also extend to humans. Some clinical trials have demonstrated effects on social cognitive outcomes. In a crossover study of children with autism spectrum disorders (ASD), 6 weeks of intranasal oxytocin treatment was found to improve parent-reported social behavior [17]. A recent 4-week crossover trial also suggests that intranasal oxytocin improves social functioning in children with ASD, with causality further supported by the observation that the effect was particularly pronounced in children with lower pretreatment peripheral oxytocin concentrations [18]. However, clinical trials in patients suffering from ASD or schizophrenia, both diseases characterized by deficits in social functioning, have produced inconsistent results [19,20]. Intranasal oxytocin therapy has also been suggested as a treatment in Prader-Willi syndrome, a complex neurodevelopmental genetic disorder characterized by a deficit in oxytocin-producing neurons in the hypothalamus, but the results so far in clinical trials with traditional spray delivery have been mixed [21,22]. Broadly speaking, these translational difficulties might be attributable to either experimental design or mechanistic issues. Regarding experimental design, the specific choice of outcome measure may contribute to disparate results, as oxytocin may only have an effect on higher order social cognition [5]. Oxytocin studies have also been statistically underpowered, in general, to detect replicable effects [23] or statistical equivalence [24] with the instruments utilized.

On a mechanistic level, translational issues have been attributed by some researchers to species differences in central oxytocin receptor distribution [25]. However, recent work suggests that humans and rodents have similar receptor distribution across the brain [26]. If central network distributions are similar, then translational failures may reflect difficulties in transporting oxytocin to the brain. Given the difficulties in transporting oxytocin across the BBB due to its molecular weight, most animal studies typically administer oxytocin via ICV injection in lieu of intravenous or oral administration. As ICV delivery is impractical for human trials, researchers have turned to intranasal nasal delivery as an apparently straightforward and inexpensive means to attempt to potentially circumvent the BBB. Despite offering several advantages, intranasal delivery is also associated with various obstacles for nasal drug uptake that may be hindering research translation, which we will discuss below.

\section{Using the nose to deliver therapeutics to the brain}

The central premises behind intranasal delivery rest on careful study of nasal cavity physiology (Figure 1) and a body of animal research that has examined the transport of other peptide molecules. Almost every human oxytocin administration study of behavior or cognition has used the intranasal route for delivery to the brain. The nasal cavity provides unique access to the brain as it is the only accessible surface of the body with direct innervation to the central compartment. The upper part of the nose is innervated with olfactory nerve endings (cranial nerve I, originating in the cerebrum) extending through the mucosa, whereas the upper and posterior regions of the nasal cavity are richly innervated by trigeminal (cranial nerve $\mathrm{V}$, originating in the brainstem) and olfactory nerve fibers (Figure 1). Intranasally delivered molecules deposited on olfactory and respiratory epithelia in this upper posterior region are then purported to travel to the CSF or directly to the central brain compartment along ensheathed channels surrounding the olfactory and trigeminal nerve fiber pathways. The delivery of oxytocin molecules to the brain via these ensheathed channels surrounding the nerve fibres is poorly characterized in man and there is no direct evidence that oxytocin molecules exploit this pathway. Moreover, the upper and posterior location of the olfactory epithelia in the nasal cavity, which is difficult to reach using conventional pump-actuated nasal spray devices, provides a physiological barrier limiting nose-to-brain transport via ensheathed channels surrounding 


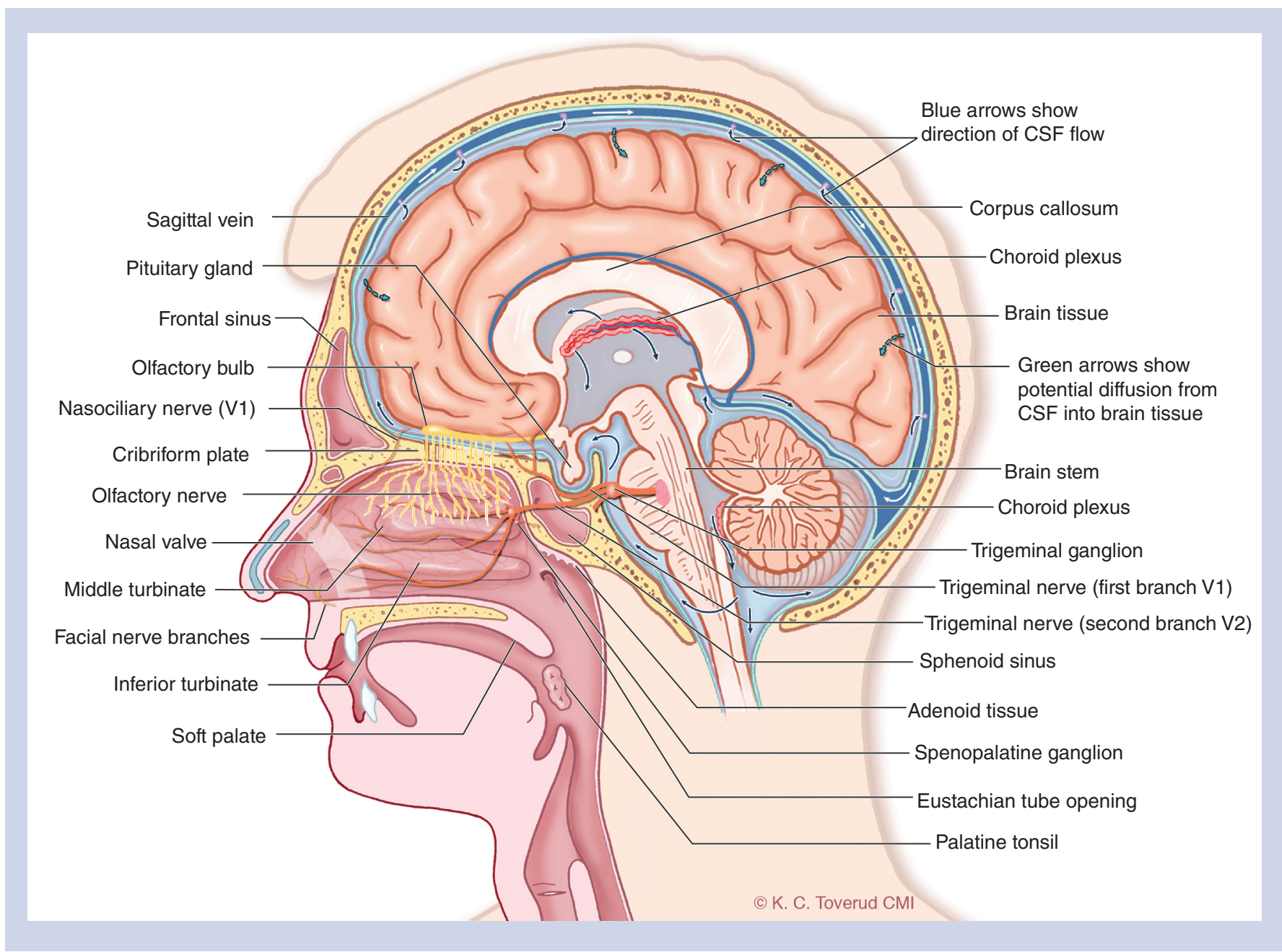

Figure 1. Key anatomical structures in the nasal cavity relevant for oxytocin transport to the brain. Reproduced with permission from [44] (c) Future Science Group (2014) and from (c) Kari C Toverud.

olfactory nerve fibers. Intranasally administered peptides are therefore in principle not only transported to the CSF, but also to the amygdala and hippocampus [27] that are rich in oxytocin receptors [26].

Olfactory receptor neurons extend to the mucous layer in the olfactory epithelium [28]. Primarily, these neurons detect odorants via odorant receptors that initiate the olfactory signaling cascade [29]. Olfactory nerve fibers traverse through small openings in the cribriform plate, pass through the subarachnoid space and terminate in the olfactory bulbs (Figure 1). Perineural channels of protective olfactory ensheathing cells, which surround bundles of approximately 1000 olfactory nerve fibers each, may offer a transport route for intranasally administered molecules as they provide an uninterrupted route from the nasal mucosa to the olfactory bulbs [30]. Research in rodents and monkeys demonstrates that molecules transported using this extracellular route take 45-90 min to appear in the olfactory bulb [31,32] and possibly much faster [33]. However, it is important to note that despite this evidence of nose-to-brain transport, less than $1 \%$ of the administered radiolabelled nerve growth factor dose appears to reach the brain [33], which is a similar response to the peripheral administration of a high oxytocin dose [7]. Moreover, the intranasal administration of arginine vasopressin, which is structurally similar to oxytocin, does not appear to reach the CSF intact, at least in dogs [34]. Altogether, these results have contributed to doubts that oxytocin intranasally delivered using conventional methods engenders functionally relevant increases of oxytocin in the brain [35]. Intranasally delivered molecules may also travel to the brain via intracellular mechanisms, but this is unlikely as intracellular transport would take hours and elevated CSF levels have been reported to occur 40-75 min after molecule administration [36,37]. Intranasal administration of wheat germ agglutinin-horseradish peroxidase 
(WGA-HRP), which at 40,000 daltons has considerably more mass than oxytocin (1007 daltons), is transported to the olfactory bulb in rats after intranasal administration [38]. Labeled molecules were also found in the olfactory epithelium and olfactory nerve layers, suggestive of transport via olfactory nerve fibers. As WGA-HRP was not meaningfully detected after intravenous administration, this supports direct nose-to-brain travel via olfactory nerve fibers. However, unlike oxytocin, WGA-HRP is a highly charged protein, which may aid nerve fiber transport.

The nasociliary branch of the ophthalmic division of the trigeminal nerve, which originates at primary trigeminal sensory nuclei via the superior orbital fissure, innervates the nasal cavity [39] (Figure 1). Some trigeminal ganglion cells with endings in the nasal epithelium also reach into the olfactory bulb via the cribiform plate [40]. After intranasal administration of labelled Insulin-like growth factor-I in rats, traces of the label are found in both the olfactory bulbs and the brainstem [41]. Intranasal administration of hypocretin-1 is also detected in the trigeminal nerve [42].

\section{Evidence for intranasal administration for central oxytocin delivery in humans}

Despite controversies regarding nose-to-brain transportation of oxytocin [35], there is evidence for nose-to-brain transport in animal studies of molecules with similar properties [43,44]. The investigation of the transportation of oxytocin molecules from the nose to the central nervous system has been hampered by the lack of an oxytocin radiotracer. However, other less direct methods provide support for nose-to-brain delivery. While brain imaging studies [45-47] can be offered as evidence for nose-to-brain oxytocin effects, such studies only indicate that intranasal oxytocin can modulate neural activity, not that this change in activity occurred via transport of the molecule from the nose to the brain. Therefore, other approaches are needed. There are two plausible alternatives for direct nose-to-brain delivery. First, intranasal oxytocin, which raises peripherally circulating levels of oxytocin [37,48-50], may cross the BBB in small amounts [7], but these quantities may still be biologically relevant [51]. If this is the case, then intravenous administration should also demonstrate effects. Indeed, early evidence that has yet to be replicated suggests that intravenous oxytocin reduces repetitive behaviors [52] and improves memory for social stimuli in ASD [53]. The second alternative to nose-to-brain delivery that would explain central activity is that intranasal oxytocin delivered to peripheral circulation leads to afferent feedback to the brain from peripheral organs rich in oxytocin receptors [54,55]. For instance, oxytocin receptors in the GI tract [56,57] or the cardiovascular system [58] may provide feedback to the brain.

Research has shown that oxytocin concentrations in CSF increase after intranasal administration in rodents [59], macaques $[36,60,61]$, and humans [37]. Increases in CSF oxytocin concentrations after intranasal oxytocin appear to be accompanied by concomitant increases in peripheral levels [62]. A study examining the dose-dependent effects of intranasal and intravenous oxytocin administration on CSF oxytocin levels in macaques found only the highest intranasal dose $(5 \mathrm{IU} / \mathrm{kg})$ increased CSF oxytocin levels, despite no increase in plasma oxytocin levels [63].

To directly examine whether intranasally administered oxytocin crosses the BBB from the peripheral circulation, researchers can compare behavioral responses between centrally targeted administration and peripheral administration. For instance, Williams and colleagues [9] examined partner preference in prairie voles after administering oxytocin both centrally and peripherally, reporting that only central administration facilitated partner preference. Regarding humans, we recently compared the social cognitive and neural effects of single-dose intranasal administration (8 and $24 \mathrm{IU}$ doses), intravenous oxytocin (1 IU), and placebo using a double-dummy crossover design. That is, participants were administered each treatment during four separate visits and at each visit, participants were administered an intranasal and intravenous solution, with solution contents determined by randomization. Despite both intravenous and intranasal oxytocin eliciting comparable peripheral concentrations after administration, only the $8 \mathrm{IU}$ intranasal dose modulated social cognition [50] and dampened right amygdala response to angry faces [45] (Figure 2) compared with placebo administration. While there were no direct measures of central oxytocin concentration, these results suggest that intranasal administration appears to elicit disproportionate behavioral and neural effects when compared with intravenous administration. If the primary activity of oxytocin were a consequence of crossing the BBB from peripheral circulation, then similar behavioral and neural effects should have been seen after IV oxytocin administration. The comparable peripheral oxytocin concentrations following IN and IV administration helped largely rule out the notion that the central effects were mediated via effects on peripheral organs which elicited afferent feedback. However, future work should also consider blocking potential peripheral actions of oxytocin with antagonists [35]. 


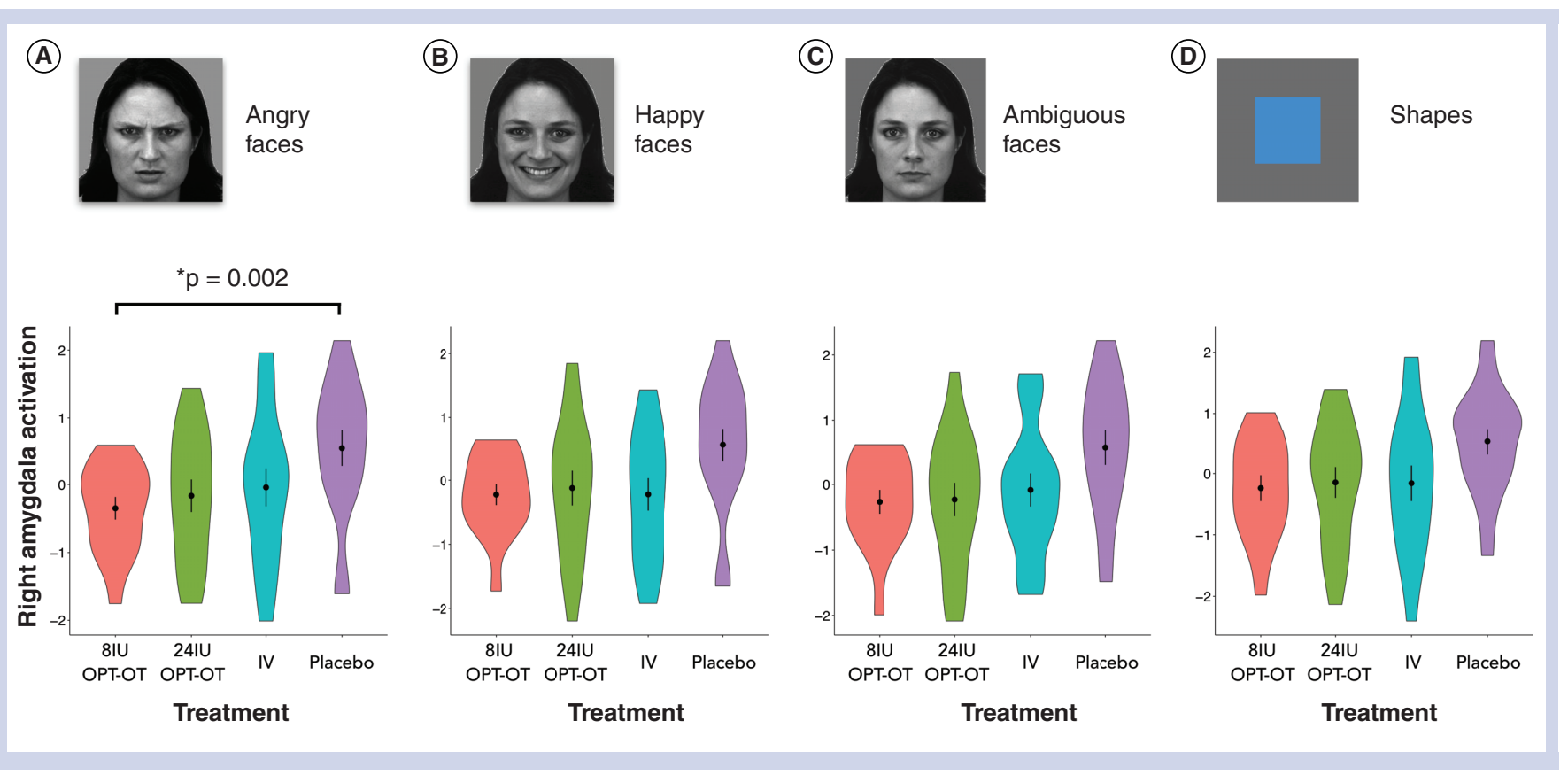

Figure 2. Dampened amygdala response to emotional stimuli after intranasal oxytocin. Right amygdala activity ( $z$-scores) during the presentation of angry faces (A), happy faces (B), ambiguous faces (C) and shapes (D). Right amygdala activity was reduced after the administration of $8 \mathrm{IU}$ oxytocin in comparison with placebo during the presentation of angry faces (A). Violin plots illustrate the distribution of the z-score normalized data by showing the probability density of the data at different values (plot tails trimmed to the range of data) with means and standard errors. ${ }^{*} p=0.002(q=0.05$, revised FDR threshold value of $p=0.008)$.

Reproduced with permission from [45] (c) Elsevier (2016).

\section{Dosage}

Most human oxytocin studies use a 24 IU dose despite very few dose ranging studies to determine the optimal dose. The use of this dose is not surprising - if prior studies found an effect using this dose why use something different? There is evidence for a modest, yet significant effect on aspects of social cognition with 24 IU using a traditional nasal spray pump for drug delivery [4]. However, lower doses may offer a stronger and more consistent response $[11,12,64,65]$. Our own dose ranging study comparing two doses (8 and $24 \mathrm{IU}$ ) found the lower dose was more efficacious in eliciting neural [45] and behavioral [50] responses, which may be explained by vasopressin cross-reactivity [51]. We observed similar outcomes in a population of adults with ASD, where a lower 8 IU dose (but not a 24 IU dose) increased emotion salience compared with placebo [66]. It is not clear why there was no response with $24 \mathrm{IU}$ in this study; however, the use of a novel exhalation delivery system (EDS) for administration of oxytocin (more details below), an approach designed to improve nasal deposition in the target regions essential for nose-to-brain transport, may have contributed to this. Further research is needed to determine the most efficacious dose in humans, and to which extent the delivery system influences dose versus response.

\section{Nasal spray formulation \& volume}

There are several unresolved issues regarding nasal spray formulation and volume that may contribute to conflicting intranasal oxytocin clinical trial data. For instance, nasal spray physicochemical properties such as viscosity [67], liposolubility, and ionasation [68], have been shown to impact spray deposition and uptake. The physicochemical properties of oxytocin and its nasal spray excipients are not well understood. Physicochemical optimization of the oxytocin nasal spray solution (e.g., permeation enhancers [68]) might improve transport kinetics through the nasal epithelium and neural sheath. If lower oxytocin doses are more likely to elicit effects $[45,50,66]$, increasing the potency of oxytocin nasal spray may also improve response, as there are physiological limits to the how much liquid the nasal cavity can absorb. 


\section{Delivery device \& deposition}

Site of deposition in the nose may significantly influence the efficiency of nose-to-brain delivery and the proportions of the drug transported to the brain versus absorbed systemically. For instance, when an angiotensin antagonist drug was delivered by pipette to the olfactory region of rats $(15 \mathrm{~mm}$ into the nasal cavity), twice the amount of drug was detected in the brain as compared with when the drug was deposited in the anterior respiratory region, which is only $7 \mathrm{~mm}$ into the nasal cavity [69]. The olfactory region of humans compared with most other mammals is substantially smaller in relation to body weight [70] and more difficult to reach for reasons including the nasal valve barrier [44]. The prime function of the nose is to filter, humidify, and warm inspired air before inhalation to the delicate lining of the lungs [71]. The anterior narrow triangular shaped valve and the complex slender nasal passages of the nasal labyrinth provide the aerodynamic features required to fulfil these important physiological functions [72]. Traditional devices used to deliver drugs intranasally, such as mechanical spray pumps and propellant driven metered-dose inhalers, deliver the majority of the drug anterior to the nasal valve [73]. The fraction that passes the valve mainly travels along the floor of the nose (Figure 1), leaving the olfactory region and large parts of the respiratory mucosa untouched [73]. Indeed, Guastella and colleagues concluded that conventional mechanical spray pumps were poorly suited to provide the delivery efficiency required for efficient and reliable nose-to-brain delivery [74]. In contrast, EDS devices significantly enhance delivery beyond the nasal valve to the upper posterior nasal segments innervated by the olfactory nerve and broadly to the respiratory mucosa with sensory innervation from the trigeminal nerve [75]. The EDS device consists of a mouthpiece and a sealing nosepiece that stents opens the valve region. When the user exhales into the mouthpiece, the soft palate elevates to seal off the nasal cavity from the oral cavity. The administered drug is released concurrently with the positive air pressure expanding the valve region, and the airflow helps carry drug deeply and broadly into target regions beyond the nasal valve. There is evidence to suggest that this approach to drug delivery may facilitate nose-to-brain drug activity. For example, a product using an EDS to deliver sumatriptan, and shown to have different pharmacokinetics than the same drug delivered via traditional spray pump, appears to produce clinical effects that are disproportionate to expectations based on only the blood pharmacokinetic profile, suggesting that part of the clinical response in the treatment of migraine is related to direct neurochemical effects [76]. In another study, this time with the easily absorbed sedative midazolam, EDS delivery of the molecule was associated with faster and more extensive sedation, similar to IV delivery that had much higher serum levels [77]. A unique nosepiece that may further optimize drug deposition after EDS delivery in the upper segments innervated by the olfactory nerve was used in the oxytocin studies described above $[45,50,66]$.

\section{Future perspective}

Successful radiolabeling of oxytocin or the development of specific ligands binding to oxytocin receptors approved for human use would be important steps to facilitate progress in the study of nose-to-brain drug transport. The inclusion of IV comparators, when the study design allows, will expand our knowledge of oxytocin delivery to the brain or at least direct activity not attributable to serum levels. Research suggests an important role for the method of drug delivery on the effect produced and on dose and, possibly, dosing frequency. If specific brain regions are the target for the nasally delivered oxytocin, and if off-target peripheral activity may produce antagonistic effects, it may be crucial to optimize direct nose-to-brain transport and limit systemic absorption. Reduced systemic absorption is also important to reduce the risk of short-term adverse events and long-term side effects that can limit the usefulness of the treatment. Dose-ranging and dose-response studies to determine the optimal dose for the various potential clinical indications, carefully considering the delivery performance and efficiency of the device, are also required.

\section{Conclusion}

There is an urgent need for therapeutics that can ameliorate social dysfunction, which is a core feature of several psychiatric illnesses. Direct central delivery of oxytocin has demonstrated considerable potential in animal models for the modulation of social behavior [9-16]. Intranasal oxytocin administration has shown some promise in humans for the treatment of ASD [18], schizophrenia [78], post-traumatic stress disorder [79], alcohol dependence [80], and social anxiety [64]; however, these results have been mixed (e.g., 81,82). A better understanding of nasal physiology and the use of intranasal delivery systems designed to improve nose-to-brain delivery may provide more consistent translational outcomes for human research. With these considerations in mind, long-term intervention trials with sufficient samples sizes are required to verify the therapeutic potential of intranasal oxytocin as a treatment modality in psychiatric and neurodevelopmental disorders. 
Direct oxytocin delivery to the brain modulates social behavior in rodents

- Evidence suggests that intracerebroventricular (ICV) oxytocin delivery modulates social behavior in rodents.

- ICV administration facilitates partner preference in the monogamous prairie vole.

- Direct injection into the medial amygdala of oxytocin receptor knockout mice rescues social amnesia.

Translating animal research to human trials

- Some findings suggest that oxytocin can improve social cognition and behavior in humans, but results have been mixed.

- Translational difficulties may be due to experimental design issues or a poor understanding of oxytocin receptor distribution in the brain.

Using the nose to deliver therapeutics to the brain

- The nasal cavity may offer a noninvasive route for oxytocin delivery to the brain via olfactory and trigeminal nerve transport.

- There are several physiological barriers that require careful consideration.

Evidence for intranasal administration for central oxytocin delivery in humans

- Brain imaging studies offer some evidence that oxytocin reaches the brain via nasal delivery. However, it is possible that these changes are secondary to peripheral oxytocin receptor activity.

- Intranasal oxytocin administration increases CSF concentrations in rodents, macaques, and humans.

- After administration of both intranasal and intravenous oxytocin in rodents and humans, effects are seen only after intranasal administration.

Dosage

- There have been very few dose-ranging oxytocin studies. However, evidence suggest that a lower dose might be more efficacious.

Nasal spray formulation \& volume

- Nasal spray physicochemical properties such as viscosity, liposolubility, and ionazation, have been shown to impact spray deposition and uptake.

- Physicochemical optimization of the oxytocin nasal spray solution might improve transport kinetics through the nasal epithelium and the neural sheath.

Delivery device \& deposition

- Site of deposition in the nose may significantly influence the efficiency of nose-to-brain delivery.

- Conventional nasal spray devices are poorly suited for reliable nose-to-brain delivery of oxytocin.

- A novel exhalation delivery system can improve nose-to-brain molecule delivery.

Future perspective

- Successful radiolabeling of oxytocin or the development of specific ligands binding to oxytocin receptors are needed to better understand nose-to-brain oxytocin transport.

- More dose-ranging and dose-response studies are required to determine the optimal oxytocin dose for the various potential clinical indications.

\section{Financial \& competing interests disclosure}

DS Quintana is supported by an excellence grant from the Novo Nordisk Foundation (NNF160C0019856). DS Quintana declares no conflict of interest. OA Andreassen has received speaker's honoraria from Lundbeck for work not directly relevant to the submitted manuscript. KT Smerud is employed (and KT Smerud owner) by Smerud Medical Research International AS, a CRO receiving fees for clinical trial services from OptiNose AS. PG Djupesland is an employee of OptiNose AS, Oslo, Norway and owns stock and stock options in OptiNose.

The authors have no other relevant affiliations or financial involvement with any organization or entity with a financial interest in or financial conflict with the subject matter or materials discussed in the manuscript apart from those disclosed.

No writing assistance was utilized in the production of this manuscript.

\section{Acknowledgements}

The authors would like to thank R Mahmoud for helpful comments on an earlier version of this manuscript. 


\section{References}

Papers of special note have been highlighted as: $\bullet$ of interest; $\bullet \bullet$ of considerable interest

1. Kosfeld M, Heinrichs M, Zak PJ, Fischbacher U, Fehr E. Oxytocin increases trust in humans. Nature 435(7042), $673-676$ (2005).

2. Nave G, Camerer C, McCullough M. Does oxytocin increase trust in humans? A critical review of research. Perspect. Psychol. Sci. 10(6), 772-789 (2015).

3. Guastella AJ, MacLeod C. A critical review of the influence of oxytocin nasal spray on social cognition in humans: evidence and future directions. Horm. Behav. 61(3), 410-418 (2012).

4. Leppanen J, Ng KW, Tchanturia K, Treasure J. Meta-analysis of the effects of intranasal oxytocin on interpretation and expression of emotions. Neurosci. Biobehav. Rev. 78, 125-144 (2017).

5. Bürkner P-C, Williams DR, Simmons TC, Woolley JD. Intranasal oxytocin may improve high-level social cognition in schizophrenia, but not social cognition or neurocognition in general: a multilevel bayesian meta-analysis. Schizophr. Bull. 43(6), 1291-1303 (2017).

6. Gimpl G, Fahrenholz F. The oxytocin receptor system: structure, function, and regulation. Physiol. Rev. 81(2), 629-683 (2001).

-• A comprehensive review of the oxytocin receptor system.

7. Mens WB, Witter A, Van Wimersma Greidanus TB. Penetration of neurohypophyseal hormones from plasma into cerebrospinal fluid (CSF): half-times of disappearance of these neuropeptides from CSF. Brain Res. 262(1), 143-149 (1983).

8. Landgraf R, Neumann ID. Vasopressin and oxytocin release within the brain: a dynamic concept of multiple and variable modes of neuropeptide communication. Front. Neuroendocrinol. 25(3-4), 150-176 (2004).

9. Williams JR, Insel TR, Harbaugh CR, Carter CS. Oxytocin administered centrally facilitates formation of a partner preference in female prairie voles (Microtus ochrogaster). J. Neuroendocrinol. 6(3), 247-250 (1994).

10. Pedersen CA, Ascher JA, Monroe YL, Prange AJ. Oxytocin induces maternal behavior in virgin female rats. Science 216(4546), 648-650 (1982).

- A seminal study describing the role of oxytocin in mammalian social behavior.

11. Benelli A, Bertolini A, Poggioli R, Menozzi B, Basaglia R, Arletti R. Polymodal dose-response curve for oxytocin in the social recognition test. Neuropeptides 28(4), 251-255 (1995).

12. Popik P, Vetulani J, Van Ree JM. Low doses of oxytocin facilitate social recognition in rats. Psychopharmacology 106(1), 71-74 (1992).

13. Engelmann M, Ebner K, Wotjak CT, Landgraf R. Endogenous oxytocin is involved in short-term olfactory memory in female rats. Behav. Brain Res. 90(1), 89-94 (1998).

14. Ferguson JN, Aldag JM, Insel TR, Young LJ. Oxytocin in the medial amygdala is essential for social recognition in the mouse. J. Neurosci. 21(20), 8278-8285 (2001).

15. László $\mathrm{K}$, Kovács A, Zagoracz $\mathrm{O}$ et al. Positive reinforcing effect of oxytocin microinjection in the rat central nucleus of amygdala. Behav. Brain Res. 296, 279-285 (2016).

16. Calcagnoli F, Stubbendorff C, Meyer N, de Boer SF, Althaus M, Koolhaas JM. Oxytocin microinjected into the central amygdaloid nuclei exerts anti-aggressive effects in male rats. Neuropharmacology 90, 74-81 (2015).

17. Yatawara C, Einfeld S, Hickie I, Davenport T, Guastella A. The effect of oxytocin nasal spray on social interaction deficits observed in young children with autism: a randomized clinical crossover trial. Mol. Psychiatry 21, 1225-1231 (2016).

- A randomized clinical crossover trial demonstrating that intranasal oxytocin oxytocin led to improvements in caregiver-rated social behavior.

18. Parker KJ, Oztan O, Libove RA et al. Intranasal oxytocin treatment for social deficits and biomarkers of response in children with autism. Proc. Natl. Acad. Sci. 201705521 (2017). https://doi.org/10.1073/pnas.1705521114 (Epub ahead of print).

19. Alvares GA, Quintana DS, Whitehouse AJ. Beyond the hype and hope: critical considerations for intranasal oxytocin research in autism spectrum disorder. Autism Res. 10(1), 25-41 (2017).

20. Bradley ER, Woolley JD. Oxytocin effects in schizophrenia: reconciling mixed findings and moving forward. Neurosci. Biobehav. Rev. 80, 36-56 (2017).

21. Einfeld SL, Smith E, McGregor IS et al. A double-blind randomized controlled trial of oxytocin nasal spray in Prader Willi syndrome. American Journal of Medical Genetics Part A 164(9), 2232-2239 (2014).

22. Tauber M, Mantoulan C, Copet $\mathrm{P}$ et al. Oxytocin may be useful to increase trust in others and decrease disruptive behaviours in patients with Prader-Willi syndrome: a randomised placebo-controlled trial in 24 patients. Orphanet J. Rare Dis. 6(1), 1-6 (2011). https://doi.org/10.1186/1750-1172-6-47 (Epub ahead of print).

23. Walum H, Waldman ID, Young LJ. Statistical and methodological considerations for the interpretation of intranasal oxytocin studies. Biol. Psychiatry 79(3), 251-257 (2016).

24. Quintana DS. Revisiting non-significant effects of intranasal oxytocin using equivalence testing. Psychoneuroendocrinology 87, 127-130 (2018). 
25. Insel TR. Translating oxytocin neuroscience to the clinic: a National Institute of Mental Health perspective. Biol. Psychiatry 79(3), 153-154 (2016).

26. Quintana DS, Rokicki J, van der Meer D et al. Genetic networks of the oxytocin system in the human brain: A gene expression and large-scale fMRI meta-analysis study. Biorxiv 149526 (2017). https://doi.org/10.1101/149526 (Epub ahead of print).

27. Chen X, Fawcett J, Rahman Y, Ala T, Frey I. Delivery of nerve growth factor to the brain via the olfactory pathway. J. Alzheimers Dis. 1(1), 35-44 (1998).

28. Doty RL, Bromley SM. Chapter 7 - Cranial nerve I: olfactory nerve. In: Textbook of Clinical Neurology (Third Edition). Goetz, CG (Ed.).W.B. Saunders, Philadelphia, USA, 99-112 (2007).

29. Kaupp UB. Olfactory signalling in vertebrates and insects: differences and commonalities. Nat. Rev. Neurosci. 11(3), 188-200 (2010).

30. Field P, Li Y, Raisman G. Ensheathment of the olfactory nerves in the adult rat. J. Neurocytol. 32(3), 317-324 (2003).

31. Shipley M. Transport of molecules from nose to brain: transneuronal anterograde and retrograde labeling in the rat olfactory system by wheat germ agglutinin-horseradish peroxidase applied to the nasal epithelium. Brain Res. Bull. 15(2), 129-142 (1985).

32. Balin BJ, Broadwell RD, Salcman M, el-Kalliny M. Avenues for entry of peripherally administered protein to the central nervous system in mouse, rat, and squirrel monkey. J. Comp. Neurol. 251(2), 260-280 (1986).

33. Frey WH, Liu J, Chen X et al. Delivery of 125I-NGF to the brain via the olfactory route. Drug Delivery 4(2), 87-92 (1997).

34. Ang V, Jenkins J. Blood-cerebrospinal fluid barrier to arginine-vasopressin, desmopressin and desglycinamide arginine-vasopressin in the dog. J. Endocrinol. 93(3), 319-325 (1982).

35. Leng G, Ludwig M. Intranasal oxytocin: myths and delusions. Biol. Psychiatry 79(3), 243-250 (2016).

36. Dal Monte O, Noble PL, Turchi J, Cummins A, Averbeck BB. CSF and blood oxytocin concentration changes following intranasal delivery in Macaque. PLoS ONE 9(8), e103677 (2014). https://doi.org/10.1371/journal.pone.0103677 (Epub ahead of print).

37. Striepens N, Kendrick KM, Hanking V et al. Elevated cerebrospinal fluid and blood concentrations of oxytocin following its intranasal administration in humans. Scientific Reports 3 (2013). https://doi.org/10.1038/srep03440 (Epub ahead of print).

38. Thorne RG, Emory CR, Ala TA, Frey II WH. Quantitative analysis of the olfactory pathway for drug delivery to the brain. Brain Res. 692(1), 278-282 (1995).

39. Prasad S, Galetta S. The Trigeminal Nerve. In: Textbook of Clinical Neurology. Goetz CG (Ed). W.B. Saunders, Philadelphia, USA, 165-183 (2007).

40. Schaefer ML, Böttger B, Silver WL, Finger TE. Trigeminal collaterals in the nasal epithelium and olfactory bulb: a potential route for direct modulation of olfactory information by trigeminal stimuli. J. Comp. Neurol. 444(3), 221-226 (2002).

41. Thorne RG, Pronk GJ, Padmanabhan V, Frey I, W H. Delivery of insulin-like growth factor-I to the rat brain and spinal cord along olfactory and trigeminal pathways following intranasal administration. Neuroscience 127(2), 481-496 (2004).

-• By using gamma counting and high-resolution phosphor imaging, this study suggests that intranasally delivered insulin-like growth factor-I is delivered to the brain via olfactory olfactory and trigeminal pathways.

42. Hanson LR, Martinez P, Taheri S, Kamsheh L, Mignot E, Frey W. Intranasal administration of hypocretin 1 (orexin A) bypasses the blood-brain barrier \& targets the brain: a new strategy for the treatment of narcolepsy. Drug Delivery Technol. 4, 65-71 (2004).

43. Merkus F, van den Berg MP. Can nasal drug delivery bypass the blood-brain barrier?: questioning the direct transport theory. Drugs $R$. D. 8(3), 133-144 (2007).

44. Djupesland PG, Messina JC, Mahmoud RA. The nasal approach to delivering treatment for brain diseases: an anatomic, physiologic, and delivery technology overview. Ther. Deliv. 5(6), 709-733 (2014).

45. Quintana DS, Westlye LT, Alnæs D et al. Low dose intranasal oxytocin delivered with Breath Powered device dampens amygdala response to emotional stimuli: a peripheral effect-controlled within-subjects randomized dose-response fMRI trial Psychoneuroendocrinology 69, 180-188 (2016).

46. Bethlehem RAI, van Honk J, Auyeung B, Baron-Cohen S. Oxytocin, brain physiology, and functional connectivity: a review of intranasal oxytocin fMRI studies. Psychoneuroendocrinology 38(7), 962-974 (2013).

47. Wigton R, Jocham Radua PA, Averbeck B et al. Neurophysiological effects of acute oxytocin administration: systematic review and meta-analysis of placebo-controlled imaging studies. J. Psychiatry Neurosci. 40(1), E1-E22 (2015).

48. Gossen A, Hahn A, Westphal L et al. Oxytocin plasma concentrations after single intranasal oxytocin administration: a study in healthy men. Neuropeptides 46(5), 211-215 (2012).

49. Kirkpatrick MG, Francis SM, Lee R, de Wit H, Jacob S. Plasma oxytocin concentrations following MDMA or intranasal oxytocin in humans. Psychoneuroendocrinology 46, 23-31 (2014).

50. Quintana DS, Westlye LT, Rustan ØG et al. Low dose oxytocin delivered intranasally with breath powered device affects social-cognitive behavior: a randomized 4-way crossover trial with nasal cavity dimension assessment. Transl. Psychiatry 5(e601), 1-9 (2015).

51. Neumann ID, Landgraf R. Balance of brain oxytocin and vasopressin: implications for anxiety, depression, and social behaviors. Trends Neurosci. 35(11), 649-659 (2012). 
- A thorough review detailing how central oxytocin and vasopressin can influence social behavior.

52. Hollander E, Novotny S, Hanratty M et al. Oxytocin infusion reduces repetitive behaviors in adults with autistic and asperger's disorders. Neuropsychopharmacology 28(1), 193-198 (2003).

53. Hollander E, Bartz J, Chaplin W et al. Oxytocin increases retention of social cognition in autism. Biol. Psychiatry 61(4), 498-503 (2007).

54. Cushing BS, Carter CS. Peripheral pulses of oxytocin increase partner preferences in female, but not male, prairie voles. Horm. Behav. 37(1), 49-56 (2000).

55. Quintana DS, Alvares GA, Hickie IB, Guastella AJ. Do delivery routes of intranasally administered oxytocin account for observed effects on social cognition and behavior? A two-level model. Neurosci. Biobehav. Rev. 49, 182-192 (2015).

56. Monstein H-J, Grahn N, Truedsson M, Ohlsson B. Oxytocin and oxytocin-receptor mRNA expression in the human gastrointestinal tract: a polymerase chain reaction study. Regul. Pept. 119(1), 39-44 (2004).

57. Qin J, Feng M, Wang C, Ye Y, Wang P, Liu C. Oxytocin receptor expressed on the smooth muscle mediates the excitatory effect of oxytocin on gastric motility in rats. Neurogastroenterol. Motil. 21(4), 430-438 (2009).

58. Gutkowska J, Jankowski M. Oxytocin revisited: its role in cardiovascular regulation. J. Neuroendocrinol. 24(4), 599-608 (2012).

59. Neumann ID, Maloumby R, Beiderbeck DI, Lukas M, Landgraf R. Increased brain and plasma oxytocin after nasal and peripheral administration in rats and mice. Psychoneuroendocrinology 38(10), 1985-1993 (2013).

60. Chang SW, Barter JW, Ebitz RB, Watson KK, Platt ML. Inhaled oxytocin amplifies both vicarious reinforcement and self reinforcement in rhesus macaques (Macaca mulatta). Proc. Natl. Acad. Sci. USA 109(3), 959-964 (2012).

61. Modi ME, Connor-Stroud F, Landgraf R, Young LJ, Parr LA. Aerosolized oxytocin increases cerebrospinal fluid oxytocin in rhesus macaques. Psychoneuroendocrinology 45, 49-57 (2014).

62. Valstad M, Alvares GA, Egknud M et al. The correlation between central and peripheral oxytocin concentrations: a systematic review and meta-analysis. Neurosci. Biobehav. Rev. 78, 117-124 (2017).

63. Freeman SM, Samineni S, Allen PC et al. Plasma and CSF oxytocin levels after intranasal and intravenous oxytocin in awake macaques. Psychoneuroendocrinology 66, 185-194 (2016).

64. Hall SS, Lightbody AA, McCarthy BE, Parker KJ, Reiss AL. Effects of intranasal oxytocin on social anxiety in males with fragile X syndrome. Psychoneuroendocrinology 37(4), 509-518 (2012).

65. Cardoso C, Ellenbogen MA, Orlando MA, Bacon SL, Joober R. Intranasal oxytocin attenuates the cortisol response to physical stress: a dose-response study. Psychoneuroendocrinology 38(3), 399-407 (2013).

66. Quintana D, Westlye L, Hope S et al. Dose-dependent social-cognitive effects of intranasal oxytocin delivered with novel breath powered device in adults with autism spectrum disorder: a randomized placebo-controlled double-blind crossover trial. Transl. Psychiatry 7(5), e1136 (2017).

67. Kundoor V, Dalby RN. Effect of formulation-and administration-related variables on deposition pattern of nasal spray pumps evaluated using a nasal cast. Pharm. Res. 28(8), 1895-1904 (2011).

68. Costantino HR, Illum L, Brandt G, Johnson PH, Quay SC. Intranasal delivery: physicochemical and therapeutic aspects. Int. J. Pharm. 337(1), 1-24 (2007).

69. Charlton S, Davis S, Illum L. Nasal administration of an angiotensin antagonist in the rat model: effect of bioadhesive formulations on the distribution of drugs to the systemic and central nervous systems. Int. J. Pharm. 338(1), 94-103 (2007).

70. Landis MS, Boyden T, Pegg S. Nasal-to-CNS drug delivery: where are we now and where are we heading? An industrial perspective. Ther. Deliv. 3(2), 195-208 (2012).

71. Harkema JR. Comparative aspects of nasal airway anatomy: relevance to inhalation toxicology. Toxicol. Pathol. 19(4-1), 321-336 (1991).

72. Jones N. The nose and paranasal sinuses physiology and anatomy. Adv. Drug Deliv. Rev. 51(1), 5-19 (2001).

73. Djupesland PG. Nasal drug delivery devices: characteristics and performance in a clinical perspective - a review. Drug Deliv. Transl. Res. 3(1), 42-62 (2012).

74. Guastella AJ, Hickie IB, McGuinness MM et al. Recommendations for the standardisation of oxytocin nasal administration and guidelines for its reporting in human research. Psychoneuroendocrinology 38(5), 612-625 (2013).

75. Djupesland PG, Skretting A. Nasal deposition and clearance in man: comparison of a bidirectional powder device and a traditional liquid spray pump. J. Aerosol. Med. Pulm. Drug Deliv. 25(5), 280-289 (2012).

76. Tepper SJ, Cady RK, Silberstein S et al. AVP-825 breath-powered intranasal delivery system containing 22 mg sumatriptan powder vs $100 \mathrm{mg}$ oral sumatriptan in the acute treatment of migraines (The COMPASS Study): a comparative randomized clinical trial across multiple attacks. Headache 55(5), 621-635 (2015).

77. Dale O, Nilsen T, Loftsson T et al. Intranasal midazolam: a comparison of two delivery devices in human volunteers. J. Pharm. Pharmacol. 58(10), 1311-1318 (2006).

78. Woolley J, Chuang B, Lam O et al. Oxytocin administration enhances controlled social cognition in patients with schizophrenia. Psychoneuroendocrinology 47, 116-125 (2014). 
79. van Zuiden M, Frijling JL, Nawijn L et al. Intranasal oxytocin to prevent posttraumatic stress disorder symptoms: a randomized controlled trial in emergency department patients. Biol. Psychiatry 81(12), 1030-1040 (2017).

80. Pedersen CA, Smedley KL, Leserman J et al. Intranasal oxytocin blocks alcohol withdrawal in human subjects. Alcohol. Clin. Exp. Res. 37(3), 484-489 (2013).

81. Dadds MR, MacDonald E, Cauchi A, Williams K, Levy F, Brennan J. Nasal oxytocin for social deficits in childhood autism: a randomized controlled trial. J. Autism Dev. Disord. 44(3), 521-531 (2014).

82. Dagani J, Sisti D, Abelli M et al. Do we need oxytocin to treat schizophrenia? A randomized clinical trial. Schizophr. Res. 172(1), 158-164 (2016).

83. Djupesland PG, Messina JC, Mahmoud RA. Breath powered nasal delivery: a new route to rapid headache relief. Headache: J. Head Face Pain 53(S2), 72-84 (2013). 
